\title{
Investigation of negative sequence injection capability in H-bridge Multilevel STATCOM
}

\author{
Ehsan Behrouzian ${ }^{1}$, Massimo Bongiorno ${ }^{1}$, Hector Zelaya De La Parra ${ }^{1,2}$ \\ ${ }^{1}$ CHALMERS UNIVERSITY OF TECHNOLOGY \\ SE-412 96, Gothenburg, Sweden \\ ${ }^{2}$ ABB Corporate Research \\ SE-721 78, Västerås, Sweden \\ Tel: +46 - (31) 7721682 \\ e-mail: ehsan.behrouzian@Chalmers.se \\ URL: http://www.chalmers.se
}

\section{Keywords}

« Multilevel converters», « Reactive power», « Static Synchronous Compensator (STATCOM)».

\section{Abstract}

The aim of this paper is to investigate the ability of star- and delta-connected $\mathrm{H}$-bridge multilevel STATCOMs to exchange negative-sequence current with the grid. Zero-sequence injection is utilized for capacitor voltage balancing. It is shown that a singularity for these configurations exists, leading to limitations in the utilization of the compensator for asymmetry compensation purposes.

\section{Introduction}

The tendency in the market clearly indicates that Voltage Source Converter (VSC) based compensators will be more and more utilized in the power systems, especially in the dynamic range for industry applications. In shunt configuration (also named STATic COMpensator, STATCOM), the VSC has a smaller footprint than the equivalent thyristor-based solution (or Static Var Compensator, SVC) and smaller harmonic filters are required. The converter behaves as a current controlled voltage source instead of a controlled susceptance as is the case of the SVC and allows fast and flexible control of the voltage at the connection point.

The H-bridge multilevel converter (also called chain-link converter) is considered one of the most attractive topologies available in the market for grid applications and it is today used by several manufacturers. Since the number of modules, or cells, is proportional to the AC voltage at the connection point, the system is scalable and it can be used without a step-up transformer for different applications [1]. This topology can be either delta or star connected. The main disadvantage with modular topologies for FACTS applications is the lack of a common dc link and therefore the difficulty in transferring energy between phase legs, in particular in case of unbalanced grids. To facilitate energy exchange, the converter must be significantly over-rated in terms of current (for the delta connection) or voltage (star connection). When star connection is utilized, a zero-sequence voltage must be introduced for the floating Y-point of the converter to exchange energy between the phases when compensating unbalanced loads [2-5]. This results in the need for an increased number of series connected cells in order to realize the needed phase voltage. On the other hand, the delta configuration allows negative-sequence compensation by letting a zero-sequence current circulate in the delta, which increases the required current rating for the compensator [5-6]. The needed overratings for the converter is a function of the negative-sequence current to be injected by the converter into the grid.

The required zero-sequence voltage (or current, depending on the selected configuration) increases exponentially when the amount of positive- and negative-sequence compensation approaches the same value, which implies that there is a limit in the compensation that can be provided by the system. This singularity happens for both delta and start configuration; for the former, when equal amounts of 
positive- and negative-sequence voltages occur and for the latter with currents. This paper presents a mathematical description of this singularity; theoretical analysis is verified via simulation for both delta and star configuration.

\section{Study of Unbalance Voltage and Current Condition}

\section{Effect of unsymmetrical voltage and negative sequence current on multilevel converters}

Fig. 1 shows the line-diagram of a H-bridge multilevel converter, both in delta and star configuration.


Fig. 1: H-bridge multilevel converter. Star configuration (a), delta configuration (b).

Assuming an unbalanced condition of the grid. By denoting the subscripts " $Y$ " and " $\Delta$ " for the measured quantities of star and delta configuration, respectively, the converter voltage and current for phase $a$ can be written as

$$
\begin{aligned}
& \bar{V}_{a Y}=\bar{V}_{a Y}{ }^{+}+\bar{V}_{a Y}{ }^{-}=v^{+} e^{j \theta^{+}}+v^{-} e^{j \theta^{-}} \\
& \bar{I}_{a Y}=\bar{I}_{a Y}{ }^{+}+\bar{I}_{a Y}{ }^{-}=i^{+} e^{j \delta^{+}}+i^{-} e^{j \delta^{-}} \\
& \bar{V}_{a \Delta}=\bar{V}_{a \Delta}{ }^{+}+\bar{V}_{a \Delta}{ }^{-}=\sqrt{3} v^{+} e^{j\left(\theta^{+}+\frac{\pi}{6}\right)}+\sqrt{3} v^{-} e^{j\left(\theta^{-}-\frac{\pi}{6}\right)} \\
& \bar{I}_{a \Delta}=\bar{I}_{a \Delta}{ }^{+}+\bar{I}_{a \Delta}{ }^{-}=\frac{1}{\sqrt{3}} i^{+} e^{j\left(\delta^{+}+\frac{\pi}{6}\right)}+\frac{1}{\sqrt{3}} i^{-} e^{j\left(\delta^{-}-\frac{\pi}{6}\right)}
\end{aligned}
$$

where, using the notation in Fig.1, the superscripts "+" and "_" denotes the positive- and the negativesequence components, respectively. The terms $v^{+}, v^{-}$and $\theta^{+}, \theta^{-}$are the converter positive- and negative-sequence output voltage in terms of amplitude and phase angle, respectively, while $i^{+}, i^{-}$and $\delta^{+}, \delta^{-}$are the converter positive- and negative-sequence output current in amplitude and angle. The instantaneous active power in each phase $\left(p_{a}, p_{b}, p_{c}\right)$ can be calculated by the inner product of the phase current and voltage for each phase. The resulting power will contain both a constant $\left(p_{a v a}, p_{a v b}, p_{a v c}\right)$ and an oscillating term (denoted as $\left.p_{o s c a}, p_{o s c b}, p_{o s c c}\right)$, which oscillates at twice the grid frequency

$$
p_{i}=p_{a v i}+p_{\text {osci }} \quad i=a, b, c
$$

Observe that, since the system is not provided with an energy storage, the sum of the average terms in the phase active power is always zero. Fig. 2 shows the instantaneous active power flowing into each 
phase (top plot) and the total three phase power (bottom plot). Under balanced condition, average active power in each phase is equal to zero. Under unbalanced condition $(t>0.84)$, different average active powers will flow in the three-phase legs. This difference in phase power is due to the presence of a negative sequence current. Notice that, under unbalanced condition, the average three phase power is still zero (Fig. 2 (b)) since the same amount of average active power which is absorbed in one phase is injected in another phase. Different average active power in the phase legs will lead to a difficulty in the capacitor balancing, as it will be discussed in the next section.
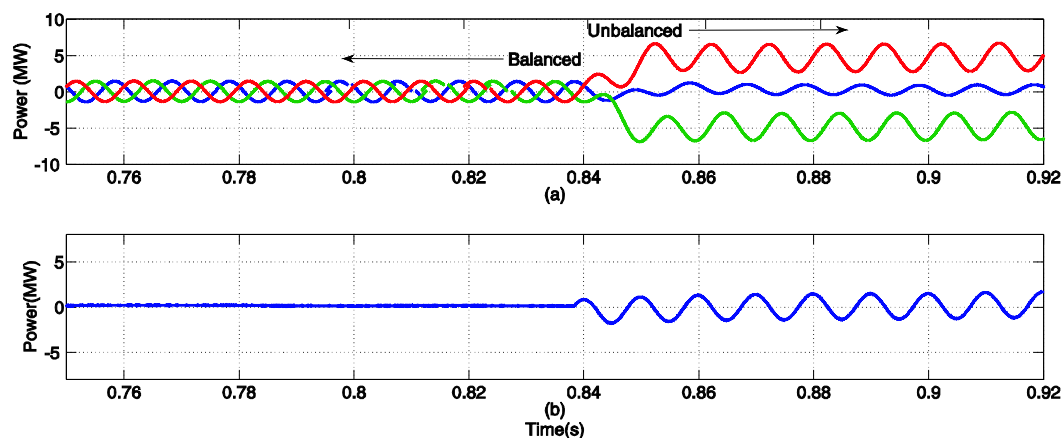

Fig. 2: Effect of unbalanced condition on active power. Each phase active power (a), three phase active power (b).

\section{Control solution for star and delta configuration under unbalance conditions}

To simplify the analysis, the converter is here considered lossless. Under this assumption, the control must guarantee a zero-average active power exchange in each phase of the converter without any grid interaction. Considering the star-connected system in Fig. 1(a), a zero-sequence voltage can be added to all phases to fulfill this requirement. Thanks to the floating neutral point of the star configuration, zero-sequence current does not flow between the converter and the grid. The applied zero-sequence voltage gives two degrees of freedom, in terms of amplitude $\left(v_{0}\right)$ and angle $\left(\theta_{v_{0}}\right)$. The goal is to find a suitable value for $v_{0}$ and $\theta_{v_{0}}$ that guarantees an average active power in each phase equal to zero. By setting the resulting average active power of two phases equal to zero, the third phase will also follow zero average active power, being the total three phase power equal to zero. Assume for example that the average active power for phases $a$ and $b$ are considered and forced to zero; in this case, the amplitude and phase of the zero-sequence voltage is summarized as [5]

$$
\begin{aligned}
& \tan \theta_{v_{0}}=\frac{k_{2} k_{3}-k_{1} k_{5}}{-k_{2} k_{4}+k_{1} k_{6}} \\
& v_{0}=\frac{-k_{1}}{k_{3} \cos \left(\theta_{v_{0}}\right)+k_{4} \sin \left(\theta_{v_{0}}\right)} \quad \text { or } \quad v_{0}=\frac{-k_{2}}{k_{5} \cos \left(\theta_{v_{0}}\right)+k_{6} \sin \left(\theta_{v_{0}}\right)}
\end{aligned}
$$

Where the constants $k_{1-6}$ are defined as

$$
\begin{aligned}
& k_{1}=\frac{1}{2} \operatorname{Re}\left[S_{a}\right]=\frac{1}{2} \operatorname{Re}\left[\bar{V}_{a Y} \bar{I}_{a Y}{ }^{*}\right] \\
& k_{2}=\frac{1}{2} \operatorname{Re}\left[S_{b}\right]=\frac{1}{2} \operatorname{Re}\left[\left(\bar{V}_{a Y}{ }^{+} e^{-j \frac{2 \pi}{3}}+\bar{V}_{a Y}{ }^{-} e^{j \frac{2 \pi}{3}}\right)\left(\bar{I}_{a Y}{ }^{+} e^{-j \frac{2 \pi}{3}}+\bar{I}_{a Y}{ }^{-j \frac{2 \pi}{3}}\right)\right] \\
& k_{3}=\frac{1}{2} \operatorname{Re}\left[\bar{I}_{a Y}\right] \\
& k_{4}=\frac{1}{2} \operatorname{Im}\left[\bar{I}_{a Y}\right] \\
& k_{5}=\frac{1}{2} \operatorname{Re}\left[\bar{I}_{b Y}\right]=\frac{1}{2} \operatorname{Re}\left[\bar{I}_{a Y}{ }^{+} e^{-j \frac{2 \pi}{3}}+\bar{I}_{a Y}{ }^{-} e^{j \frac{2 \pi}{3}}\right] \\
& k_{6}=\frac{1}{2} \operatorname{Im}\left[\bar{I}_{b Y}\right]=\frac{1}{2} \operatorname{Im}\left[\bar{I}_{a Y}{ }^{+} e^{-j \frac{2 \pi}{3}}+\bar{I}_{a Y}{ }^{-} e^{j \frac{2 \pi}{3}}\right]
\end{aligned}
$$

In delta structures (Fig. 1(b)) instead, a circulating current must be used $\left(i_{0} e^{j \delta_{i_{0}}}\right)$. The circulating current flows only inside the delta, allowing power exchange between the phases without affecting the grid.

Similar to the previous case, the zero-sequence current allows two degrees of freedom (amplitude, $i_{0}$, and angle, $\delta_{i_{0}}$, of the zero-sequence current). Following the same criteria as for star case and setting 
the active power in phases $a$ and $b$ to zero, the amplitude and phase of the zero-sequence current can be calculated as

$$
\begin{aligned}
& \tan \delta_{i_{0}}=\frac{-k_{11} k_{15}+k_{12} k_{13}}{-k_{12} k_{14}+k_{11} k_{16}} \\
& i_{0}=\frac{-k_{11}}{k_{13} \cos \left(\delta_{i_{0}}\right)+k_{14} \sin \left(\delta_{i_{0}}\right)} \quad \text { or } \quad i_{0}=\frac{-k_{12}}{k_{15} \cos \left(\delta_{i_{0}}\right)+k_{16} \sin \left(\delta_{i_{0}}\right)}
\end{aligned}
$$

where the terms $\mathrm{k}_{11-16}$ are defined as

$$
\begin{aligned}
& k_{11}=\frac{1}{2} \operatorname{Re}\left[S_{a}\right]=\frac{1}{2} \operatorname{Re}\left[\bar{V}_{a \Delta} \bar{I}_{a \Delta}{ }^{*}\right] \\
& k_{12}=\frac{1}{2} \operatorname{Re}\left[S_{b}\right]=\frac{1}{2} \operatorname{Re}\left[\left(\bar{V}_{a \Delta}{ }^{+} e^{-j \frac{2 \pi}{3}}+\bar{V}_{a \Delta}{ }^{-} e^{j \frac{2 \pi}{3}}\right)\left(\bar{I}_{a \Delta}{ }^{+} e^{-j \frac{2 \pi}{3}}+\bar{I}_{a \Delta}{ }^{-} e^{j \frac{2 \pi}{3}}\right)\right] \\
& k_{13}=\frac{1}{2} \operatorname{Re}\left[\bar{V}_{a \Delta}\right] \\
& k_{14}=\frac{1}{2} \operatorname{Im}\left[\bar{V}_{a \Delta}\right] \\
& k_{15}=\frac{1}{2} \operatorname{Re}\left[\bar{V}_{b \Delta}\right]=\frac{1}{2} \operatorname{Re}\left[\bar{V}_{a \Delta}{ }^{+} e^{-j \frac{2 \pi}{3}}+\bar{V}_{a \Delta} e^{-j \frac{2 \pi}{3}}\right] \\
& k_{16}=\frac{1}{2} \operatorname{Im}\left[\bar{V}_{b \Delta}\right]=\frac{1}{2} \operatorname{Im}\left[\bar{V}_{a \Delta}{ }^{+} e^{-j \frac{2 \pi}{3}}+\bar{V}_{a \Delta} e^{j \frac{2 \pi}{3}}\right]
\end{aligned}
$$

\section{Operating Range in Case of Negative Sequence Current Injection}

In order to simplify the analysis, the grid is considered to be balanced and only reactive power is exchanged between the grid and the converter. Therefore, voltages and currents can be written a

$$
\begin{array}{ll}
i^{+}=\frac{v^{+}-e}{X_{f}} \Rightarrow v^{+}=X_{f} i^{+}+e & , \delta^{+}=\theta^{+}-\frac{\pi}{2} \\
i^{-}=\frac{v^{-}}{X_{f}} \Rightarrow v^{-}=X_{f} i^{-} & , \delta^{-}=\theta^{-}-\frac{\pi}{2}
\end{array}
$$

where $X_{f}$ is the reactance of the filter installed between the grid and the converter, while $e$ is the positive sequence amplitude of the grid voltage. By replacing (7) into (3) to (6), the relation between $v_{0}$ and $i^{-}$for the star case and between $i_{0}$ and $v^{-}$for the delta case are obtained as

$$
v_{0}=k_{Y} i^{-}, 0<k_{Y}<\infty, i_{0}=k_{\Delta} v^{-}, 0<k_{\Delta}<\infty
$$

The terms $k_{Y}$ and $k_{\Delta}$ is (8) are not constant and are a function of the operating conditions. The solution for the voltage/current shows that a practical limitation exists in both configurations. Under specific operating conditions, the zero-sequence voltage needed for the star configuration can become infinity. Because of duality between star and delta, infinite zero-sequence current for delta configuration is also expected at the dual condition.

The operating range of star configuration is limited to the degree of unbalance $\left(i^{-} / i^{+}\right)$. From the set of equations described earlier, it can be proven that $k_{Y}$ will become infinity if $\left(i^{-} / i^{+}\right)=1$, leading to a theoretical infinite voltage for the Y-point to allow power distribution among phases in this specific case. In practice, maximum attainable output voltage of the converter leg determines additional limitations on the degree of unbalance. If the required converter voltage exceeds the maximum converter voltage rating and the converter operates in over modulation mode then dc-link voltages will be uncontrollable.

Because of duality between star and delta the same phenomena is expected for delta configuration as well. The operating range of delta configuration is limited to the degree of voltage unbalance $\left(v^{-} / v^{+}\right)$. It can be proven that $k_{\Delta}$ will become infinity if $\left(v^{-} / v^{+}\right)=1$, leading to an infinite circulating current. 
Observe that this singularity occurs if $\left|\delta^{+}-\delta^{-}\right|=\pi$ for the star or, correspondingly, $\left|\theta^{+}-\theta^{-}\right|=$ $2 \pi / 3$ for the delta configuration.

Fig. 3 shows the relation between positive- and negative-sequence voltages and zero-sequence current amplitude for delta configuration (Fig. 3(a)) and the relation between positive- and negative-sequence current and zero-sequence voltage amplitude for star configuration (Fig. 3(b)). As this figure clearly shows, infinite zero-sequence voltage (current) is needed when $\left(i^{-} / i^{+}\right)=1$ or $\left(v^{-} / v^{+}\right)=1$ respectively.

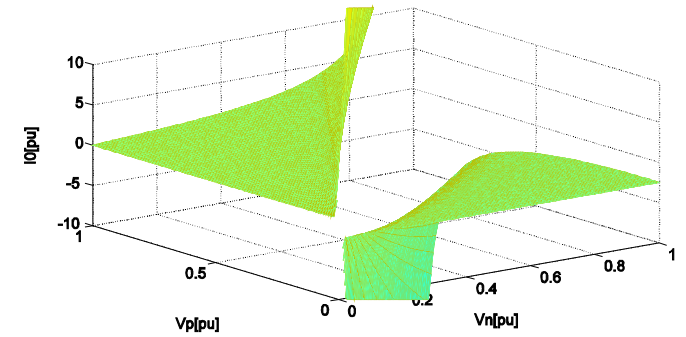

(a)

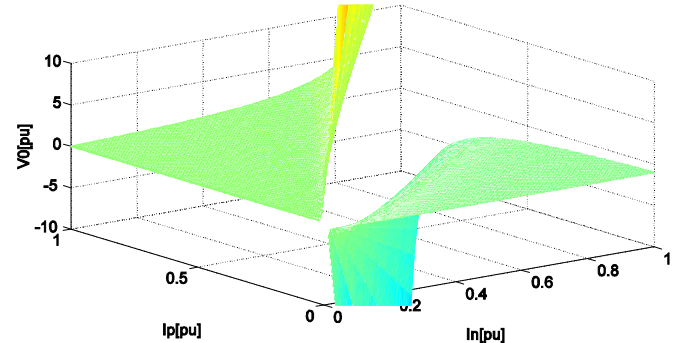

(b)

Fig. 3: Relationship between zero sequence current and positive and negative sequence voltage for delta configuration (a) and between zero sequence voltage and positive and negative sequence currents for star configuration (b).

\section{Control of H-bridge Multilevel STATCOM Under Unbalance Condition}

In the previous chapter, zero-sequence voltage and current have been calculated for a lossless converter. It is also possible to calculate these parameters when average active power in each phase is not equal to zero due to losses. Considering the average active power of phase $a, b, c$ as $p_{a}, p_{b}, p_{c}$, zero sequence voltage and current can be calculated as

$$
\begin{aligned}
& \tan \theta_{v_{0}}=\frac{-\left(\left(p_{b}-k_{2}\right) k_{3}-\left(p_{a}-k_{1}\right) k_{5}\right)}{\left(p_{b}-k_{2}\right) k_{4}-\left(p_{a}-k_{1}\right) k_{6}} \\
& v_{0}=\frac{p_{a}-k_{1}}{k_{3} \cos \left(\theta_{v_{0}}\right)+k_{4} \sin \left(\theta_{v_{0}}\right)}=\frac{p_{b}-k_{2}}{k_{5} \cos \left(\theta_{v_{0}}\right)+k_{6} \sin \left(\theta_{v_{0}}\right)} \\
& \tan \delta_{i_{0}}=\frac{\left(P_{a}-k_{11}\right) k_{15}-\left(P_{b}-k_{12}\right) k_{13}}{\left(P_{b}-k_{12}\right) k_{14}-\left(P_{a}-k_{11}\right) k_{16}} \\
& i_{0}=\frac{P_{a}-k_{12}}{P_{13} \cos \left(\delta_{i_{0}}\right)+k_{14} \sin \left(\delta_{i_{0}}\right)}=\frac{P_{15} \cos \left(\delta_{i_{0}}\right)+k_{16} \sin \left(\delta_{i_{0}}\right)}{k_{1}}
\end{aligned}
$$

In this section, the control algorithm of the H-bridge multilevel STATCOM under unbalance condition will be presented. The goal is to independently control both positive- and negative-sequence currents injected to the grid, and keep all capacitor voltages balanced at the desired value. Fig. 4 shows the control block diagram for both star and delta connection.

In the following, the Delayed Signal Cancellation (DSC), the Dual Vector Current Control (DVCC), the zero sequence control for cluster controlling (defined as average voltage of all dc link voltages in each phase) and the individual capacitor voltage balancing control (defined as dc-link voltage of each cell) are explained.

\section{Delayed Signal Cancellation (DSC)}

To be able to control the current also during unbalance condition, an algorithm for estimation of positive- and negative-sequence components is needed. This algorithm is here called Delayed Signal Cancellation (DSC).

Any unbalance voltage or current can be written as 


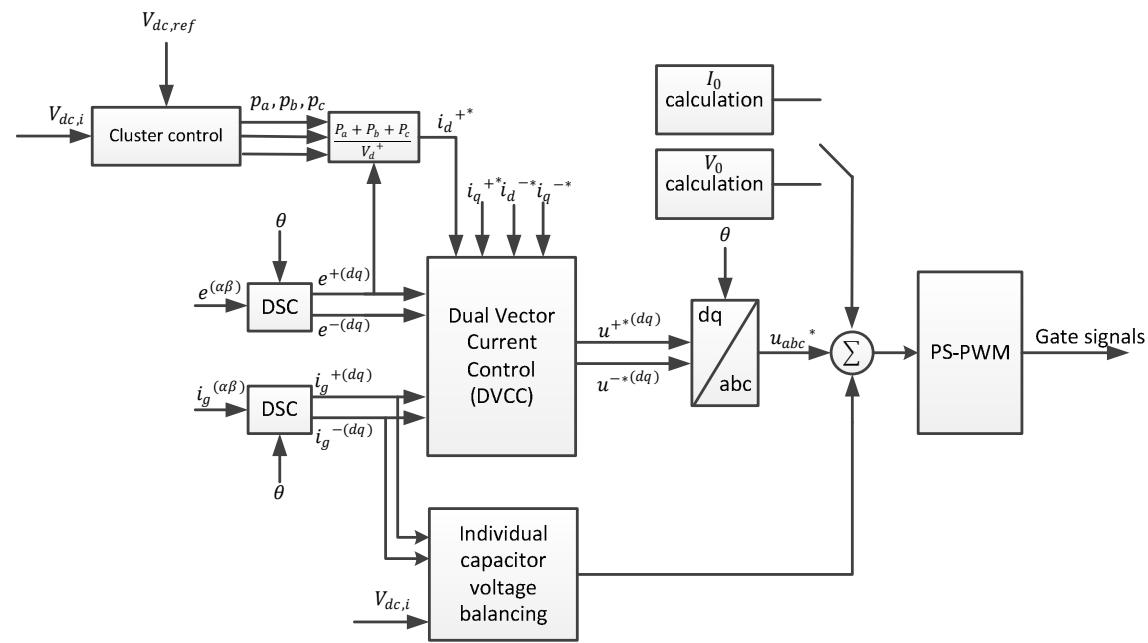

Fig .4: Control block diagram of H-bridge multilevel STATCOM for unbalance condition. Zero-sequence voltage for star and zero-sequence current for delta structure.

$e^{(\alpha \beta)}(t)=e^{+(\alpha \beta)}(t)+e^{-(\alpha \beta)}(t)$

where $e^{+(\alpha \beta)}$ is the vector of the positive and $e^{-(\alpha \beta)}$ is the vector of the negative phase-sequences of the voltage or current.

In the fixed $\alpha \beta$-coordinate system, the positive phase-sequence vector rotates counterclockwise, while the negative rotates with the same frequency clockwise. Delaying the voltage or currents with vectors $\underline{e}^{(\alpha \beta)}(t)$ by a quarter of cycle yields

$\underline{e}^{+(\alpha \beta)}=j \underline{e}^{+(\alpha \beta)}\left(t-\frac{T}{4}\right)$

$\underline{e}^{-(\alpha \beta)}=-j \underline{e}^{-(\alpha \beta)}\left(t-\frac{T}{4}\right)$

(11) can be written as

$j e^{(\alpha \beta)}\left(t-\frac{T}{4}\right)=e^{+(\alpha \beta)}(t)\left(t-\frac{T}{4}\right)+j e^{-(\alpha \beta)}(t)\left(t-\frac{T}{4}\right)$

Adding (11) and (14) yields

$e^{(\alpha \beta)}(t)+j e^{(\alpha \beta)}\left(t-\frac{T}{4}\right)=e^{+(\alpha \beta)}(t)+e^{+(\alpha \beta)}\left(t-\frac{T}{4}\right)+e^{-(\alpha \beta)}(t)+j e^{-(\alpha \beta)}\left(t-\frac{T}{4}\right)$

Using (12) and (13) and simplifying (15) yields

$e^{+(\alpha \beta)}(t)=\frac{1}{2}\left(e^{(\alpha \beta)}(t)+j e^{(\alpha \beta)}\left(t-\frac{T}{4}\right)\right)$

The delayed signal cancellation (DSC) method for sequence detection is defined by

$\underline{\hat{e}}^{+(\alpha \beta)}(t)=\frac{1}{2}\left(e^{(\alpha \beta)}(t)+j e^{(\alpha \beta)}\left(t-\frac{T}{4}\right)\right)$

$\underline{\hat{e}}^{-(\alpha \beta)}(t)=\frac{1}{2}\left(e^{(\alpha \beta)}(t)-j e^{(\alpha \beta)}\left(t-\frac{T}{4}\right)\right)$

where $\underline{\hat{e}}^{+(\alpha \beta)}(t)$ and $\underline{\hat{e}}^{-(\alpha \beta)}(t)$ are the estimated positive and negative sequence components of the voltages or currents and $\mathrm{T}$ is the period of these waveforms. 
Fig. 5 displays the block diagram of DSC applied in discrete time. The term " $d q^{+}$" denotes a $d q$-plane that rotates counterclockwise with the angular frequency of the system, while the term " $d q^{-}$" denotes a $d q$-plane that rotates clockwise with the angular frequency. These two planes can also be called positive and negative synchronous reference frames (DRFs). $\theta_{(k)}$ is the output of the Phase-Locked Loop (PLL).

When using a discrete controller, the measured signal $\underline{e}^{(\alpha \beta)}(k)$ has to be delayed of $f_{s} / 4 f_{g}$ samples which should be an integer for a perfect estimation. $f_{s}$ is the sampling frequency and $f_{g}$ is the voltage or current frequency.



Fig .5: Block diagram of DSC technique implemented in discrete-time domain.

\section{Dual Vector Current-Controller (DVCC)}

The vector current controller must be able to control both positive- and negative- sequence components of the filter current independently. To achieve this goal, a Dual Vector Current Controller (DVCC) can be used.

The DVCC is constituted by two separate controllers implemented in the positive and in the negative synchronous reference frame, respectively, as shown in Fig. 6. Both grid voltage and current are separated into positive- and negative-sequence components and the controller tracks positive- and negative-sequence reference currents. CC in Fig. 6 denotes the vector current control.

The DVCC outputs are positive- and negative-sequence components of the references voltage for the PWM modulator in the two synchronous reference frames. By transforming them into the fixed $\alpha \beta$ coordinate system and then adding them up the reference voltage vector $\underline{u}^{(\alpha \beta) *}$ is obtained.

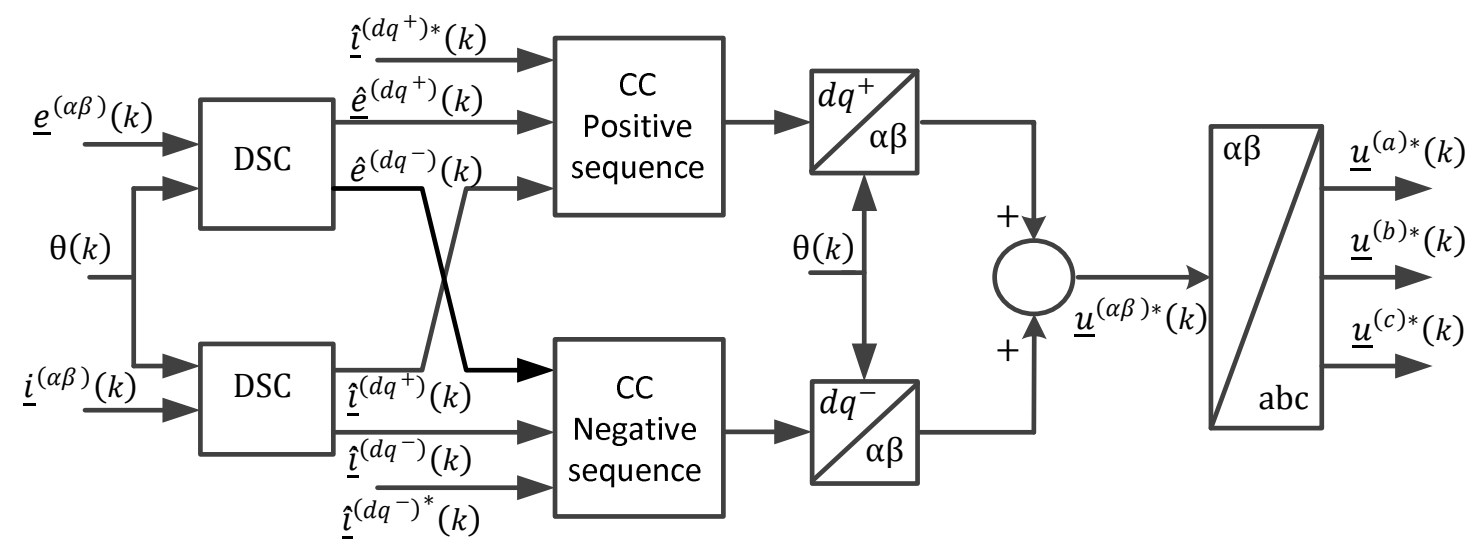

Fig.6: Block diagram of dual vector current-controller (DVCC).

\section{Cluster control, zero-sequence voltage and zero sequence current}

Cluster voltage for phase $a$ defines as

$V_{a}=\frac{v_{c a 1}+v_{c a 2}+\cdots+v_{c a n}}{n}$

where $v_{c a 1}$ to $v_{c a n}$ are the dc link voltages of phase $a$ and $n$ is the number of cells in each phase. 
Fig. 7 shows the cluster voltage controller. Here we control the energy stored in the dc caps. Outputs of the PI controllers are the reference active powers for each phase. The amplitude of the active current is obtained by

$$
\begin{aligned}
& P_{t}=P_{a}+P_{b}+P_{c} \\
& I_{d}{ }^{*}=\frac{P_{t}}{V_{d}{ }^{+}}
\end{aligned}
$$

$V_{d}{ }^{+}$is the grid $d$-component positive-sequence voltage. $I_{d}{ }^{+}$provides the total active power needed to charge all the capacitors in three phases. The generated reference active power $P_{a}, P_{b}$ are used to calculate required zero-sequence voltage or current (see (9) and (10)).



Fig .7: Block diagram of cluster controller.

In order to calculate zero sequences, positive- and negative-sequences amplitude and phase of the converter voltages and currents should be measured first. Following equations can be used to calculate these values.

$$
\begin{array}{ll}
v^{+}=\frac{3 \sqrt{2}}{\sqrt{3}} \cdot \sqrt{V_{d}^{+* 2}+V_{q}^{+* 2}}, & \theta^{+}=\tan ^{-1}\left(\frac{V_{d}^{+*}}{V_{q}^{+*}}\right) \\
v^{-}=\frac{3 \sqrt{2}}{\sqrt{3}} \cdot \sqrt{V_{d}^{-* 2}+V_{q}^{-* 2}}, & \theta^{-}=\tan ^{-1}\left(\frac{V_{d}^{-*}}{V_{q}^{-*}}\right) \\
i^{+}=\frac{3 \sqrt{2}}{\sqrt{3}} \cdot \sqrt{i_{d}^{+2}+i_{q}^{+2}}, & \delta^{+}=\tan ^{-1}\left(\frac{i_{q}^{+}}{i_{d}^{+}}\right) \\
i^{-}=\frac{3 \sqrt{2}}{\sqrt{3}} \cdot \sqrt{i_{d}^{-2}+i_{q}^{-2}} & , \quad \delta^{-}=\tan ^{-1}\left(\frac{i_{q}^{-}}{i_{d}^{-}}\right)
\end{array}
$$

$V_{d}^{+*}, V_{q}^{+*}$ and $V_{d}^{-*}, V_{q}^{-*}$ are positive and negative sequence reference voltages respectively. $i_{d}^{+}, i_{q}^{+}$and $i_{d}^{-}, i_{q}^{-}$are positive and negative sequence currents respectively.

\section{Individual capacitor voltage balancing control}

The purpose of the individual balancing controller is to modify the modulation index for each cell in order to keep the dc-link voltages balanced. For this purpose, a sinusoidal voltage signal is added to the modulation index of each cell. The amplitude of this voltage is proportional to the voltage error ( $\Delta v_{c 1}$ for example) and it is in phase with the phase current.

In case of unbalance condition and by assuming $i_{q} \gg i_{d}$, the phase $a$ current for star connection is

$i_{a}=\sqrt{\frac{2}{3}} I_{q}^{+} \sin (\theta)+\sqrt{\frac{2}{3}} I_{q}^{-} \sin (-\theta)$

And for delta connection

$i_{a}=\frac{\sqrt{2}}{3} I_{q}^{+} \sin (\theta+\pi / 6)+\frac{\sqrt{2}}{3} I_{q}^{-} \sin (\theta-\pi / 6)$ 
Instead of positive and negative reactive current, the reference values for these two parameters can be used. This can be done assuming the fact that the current controller tracks the reference values, and its dynamic is much faster than the individual controller dynamic. Since the measured $I_{q}^{+}$and $I_{q}^{-}$contains ripple and distortion, replacing the real values by the reference values leads to producing the voltage commands without distortion. The voltage signal for the cells in phase $a$ are as

$v_{a 1}=K_{a}\left(V_{a}-v_{c 1}\right) \cdot i_{a}, v_{a 2}=K_{a}\left(V_{a}-v_{c 2}\right) \cdot i_{a}, \ldots, v_{a n}=K_{a}\left(V_{a}-v_{c n}\right) \cdot i_{a}$

$K_{a}$ is the proportional gain of the individual balancing controller. Analogously, the voltage signal for each cell in phase $b$ and phase $c$ can be obtained.

The calculated zero-sequence voltage together with the signal output of the individual voltage balancing will be added to the references voltages provided by the DVCC. The final voltage reference for each cell is normalized by the capacitor voltage of that cell to generate the modulation index of the cell. Finally Phase-Shift PWM provides the switching pattern for the converter.

\section{Simulation Results}

A 120MVA STATCOM with delta-connected configuration as shown in Fig. 1(b), with three cells per phase is simulated under unbalanced condition. Fig. 8(a) shows the degree of unbalance $\left(v^{-} / v^{+}\right)$. Fig. 8(b) shows the circulating current. As expected, increasing the $\left(v^{-} / v^{+}\right)$leads to an increase in circulating current amplitude. $\left(v^{-} / v^{+}\right)=1$ is the critical point. At around $\left(v^{-} / v^{+}\right)=0.7$ a circulating current with nearly $0.6 \mathrm{pu}$ is needed. Fig. 8(c) shows the three-phase grid voltage, while Fig. 8(d) shows the average active power in each phase leg. It clearly shows how circulating current can manage to control the average active power in each phase. Fig. 8(e) shows $k_{\Delta}$ factor versus $\left(v^{-} / v^{+}\right)$. This simulation results prove the fact that delta configuration is sensitive to $\left(v^{-} / v^{+}\right)$and more than $1 \mathrm{pu}$ circulating currents is needed for $\left(v^{-} / v^{+}\right)$higher than 0.7 .

Fig. 9 shows the obtained simulation results when star configuration, as shown in Fig. 1(a), is used instead. STATCOM rating and number of cells are the same as delta configuration. Fig. 9(a) shows the degree of unbalance $\left(i^{-} / i^{+}\right)$. Fig. 9(b) shows the zero sequence voltage. As expected, increasing the $\left(i^{-} / i^{+}\right)$leads to an increase in zero sequence voltage amplitude. $\left(i^{-} / i^{+}\right)=1$ is the critical point. At around $\left(i^{-} / i^{+}\right)=0.7$ a zero sequence voltage with nearly $1 \mathrm{pu}$ is needed. Fig. 9(c) shows the three phase injected current to the grid. Fig. 9(d) shows the average active power in each phase leg. It clearly shows how zero sequence voltage can manage to control the average active power in each phase. Fig. 9 (e) shows $k_{y}$ factor versus $\left(i^{-} / i^{+}\right)$. This simulation results prove the fact that star configuration is sensitive to $\left(i^{i^{-}} / i^{+}\right)$and more than $1 \mathrm{pu}$ zero sequence voltages are needed for $\left(i^{-} / i^{+}\right)$higher than 0.7 .

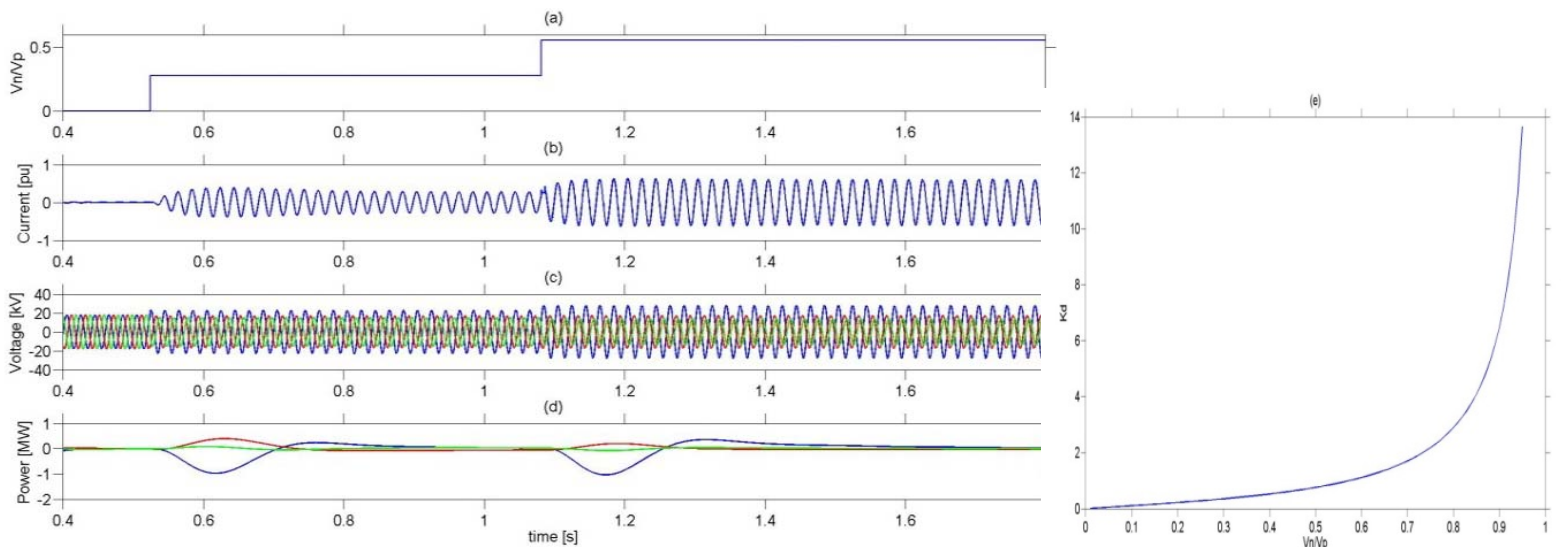

Fig. 8: Simulation results of delta configuration. $\frac{v^{-}}{v^{+}}(\mathrm{a})$, circulating current (b), 3-phase voltages (c), average active power (d), $k_{\Delta}(\mathrm{e})$. Base current is $1 \mathrm{kA}$. 

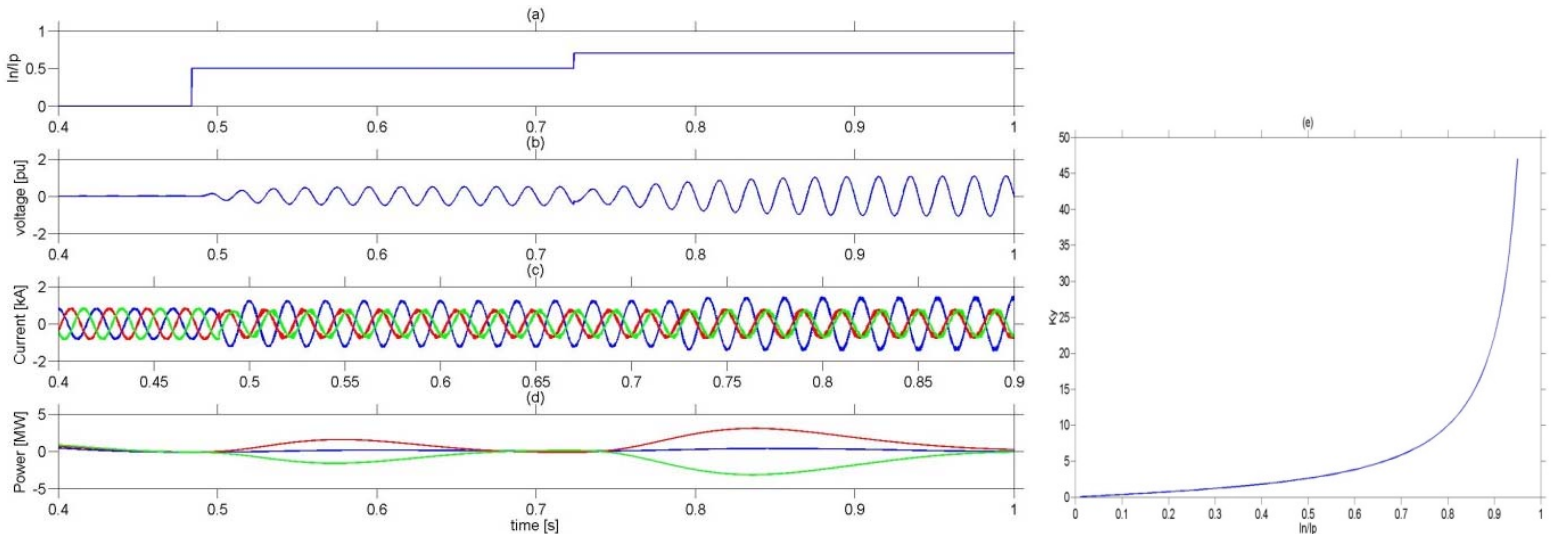

Fig. 9: Simulation results of star configuration. $\frac{i^{-}}{i^{+}}$(a), zero sequence voltage (b), 3-phase injected current (c), average active power (d), $k_{Y}(\mathrm{e})$. Base voltage is $33 \mathrm{kV}$

\section{Conclusion}

In this paper, the effect of unsymmetrical voltage and current on H-Bridge chain link converters has been investigated, both in case of star and delta connection of the converter phase legs. Zero-sequence voltage (for the star configuration) or current (delta) allow maintaining the dc link of the different cells balanced also in case of negative-sequence current exchange between the converter and the grid. However, it has been shown that there are special operating conditions for both star and delta where zero-sequence voltage/current are unable to control the average active power in each phase to zero. This is due to a singularity that exists in the solution for the calculation of the zero-sequence components. It is of importance to stress that although this singularity exists both for the start and for the delta configuration, the probability of encountering the critical operating point for the delta case is much less than for the star case. This is due to the fact that the singularity in the delta configuration is governed by the equality between positive and negative sequence voltage, while for the star case it is governed by the equality between positive and negative sequence current. Being the grid voltage mainly a positive sequence (also under unbalanced conditions), the singularity for the delta configuration has fewer probabilities to occur as compared with the star one. This can be a strong reason to choose delta configuration instead of star when negative sequence control is required by the customer.

\section{References}

[1] Akagi H, Inoue S, Yoshii T .: Control and Performance of a Transformer less Cascade PWM STATCOM With Star Configuration, IEEE Transactions on Power Systems, Vol. 43 no 4, pp. 1041 - 1049, Aug. 2007.

[2] Betz R.E, Summerst T, Furneyt T.: Symmetry Compensation using a H-Bridge Multilevel STATCOM with Zero Sequence Injection, IEEE Conference, pp. 1724 - 1731, 2006.

[3] Song Q, Liu W.: Control of a Cascade STATCOM with Star Configuration under Unbalanced Conditions, IEEE Transactions on Power electronics, Vol. 24 no 1, pp. 45 - 58, Jan. 2009.

[4] Hatano N, Ise T.: Control Scheme of Cascaded H-Bridge STATCOM Using Zero-Sequence Voltage and Negative-Sequence Current, IEEE Transaction on power delivery, Vol. 25 no 2, pp. 543 - 550, Apr. 2010.

[5] Betz R, Summers T.: Introduction to Symmetrical Components and their use in STATCOM Applications, ResTech Pty Ltd School of Electrical Engineering and Computer Science University of Newcastle, Australia 2009.

[6] Hagiwara M, Maeda R, Akagi H .: Negative-Sequence Reactive-Power Control by a PWM STATCOM Based on a Modular Multilevel Cascade Converter (MMCC-SDBC), IEEE Transactions on industry applications, Vol. 48 no 2, pp. 720 - 729, Apr. 2012. 